
QK

i 50

.965

1880

$+\cdots$,

vองาน unks

New yos:

EOTRWIGAL

G.ำ 


\section{PRELININARY LISTI}

OF THE

Plants Growing without Cultivation IIT

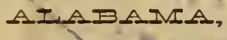

FROM THE COLLEC TIONS MADE BY

IUGENE A.SMITE, TusCaloosa, \& CEAS.MOER, Mobile,Ala.

COMPILED BY CHARLES MOHR. $18 \& 0$

[Explanation.-The letters M. (Mohr) and S. (-mith), atlixed to the names of the species, will indicate the collector.]

I. Ranunculacece.

1. Clematis Crispa, I., S., M.

Catesbyana, Pursh., Clarke

- county, M.

Viorna, L., M., Mol.

reticulata, Walt.,Cl'ke Co.M.

2. Anemone nemorosi, L., S.

Caroliniana, Walt., S.

Virginiana, L., S.

heterophylla, Nutt., S.

3. Thalictrum anemonoides, Michx., s.

debile, Buckl., Wilcox Co., ex herb., M.

4. Hepatica triloba, C'haix., S.

5. Myosurus minimus, L., S.

6. Rauunculus parvitiorus, L., S., M.

muricatus, L., M.

pusillus, Poir., M, 


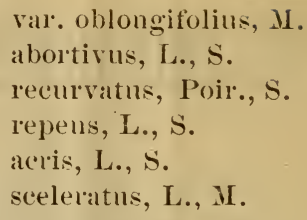

7. Isopyrum biteruatum, 'Torr, \& Gr.,s.

8. Delphinimm azureum, Michx., s., M.

$$
\begin{aligned}
& \text { tricorne, Miclux., s. } \\
& \text { exaltatum, Ait., s. }
\end{aligned}
$$

9. Aconitum uncinatum, L., S.

10. Zanthorhiza apiifolia, I'Her., S.

11. Actæa alba, Bigel, S., M.

II. Magnoliacec.

12. Illicium Floridamm, Ellis, S., M.

13. Magnolia graulitlora, L. S., M.

glamea, L., S., Ji.
Umbrella, Lam., s.
acuminata, L., s.

Fraseri, Walt., Clarke Co.,

Demuy.

macrophylla, Michx., M.

14. Liriodendron 'V'ulipfera, L., S.

\section{Anonacece.}

15. Asimina parviflora, Dumal, S. M.

IV. Menispermacec.

16. Cocculus Caroliuus, DC. S.

17. Calycocarpun Lyoni, Nutt., s.

V. Berberidacece.

18. Podophyllum peitatum, L. s. 


\section{Nelumbiacece.}

19. Nelumbium lnteum, Wild, M.

VII. Cabombacece.

20. Cabomba Caroliniana, Gray, M.

21. Brasenia peltata, Pursh, M.

\section{Nymphoacex.}

22. Nuphar adrena, Ait. S.

sagittaefolia, Pursh., S. M.

IX. Papaveracea.

23. Sanguinaria Canadensis, L., S.

24. Argemone Mexicana, L., M.

X. Sarraceniacea.

25. Sarracenia purpurea, L. II,

Psittacina, Michx., M, rubra, Walt. II.

I)rummondii, Croom, M.

flava, L. II.

XI. Fumariacea.

26. Dicentra Cucullaria, DC., S.

27. Corydalis aurea, Wild, S., M.

XII. Cruciferce.

28. Cardamine rhomboidea, DC., S.

Ludoriciana, Hook, S., M.

hirsuta, L., S.

¿9. Arabis Candensis, L. S.

laevigata, DC., S.

30. Sisymbrium Thaliana, Gaud., S., M.

31. Dentaria diphylla, Miehx., S.

laciniata, Muhl., S.

3:.). Draba cuneifolia, Nutt., S., II. 
33. Senebiera pinnatifida, DC., S.

34. Capsella Bursa-pastoris, Moench., S M.

35. Lepidium Virginicum, L., S., M.

36. Cakile maritima, Scop.

Var. aequalis, Chap., M.

\section{Capparidacea.}

37. Polanisia graveolens, M.

38. Gynandropsis pentaphylla, DC., S.

XIV. Violace $\alpha$.

39. Viola cucullata, Ait., S., M.

palmata, L. S., M.

var. septemloba, Le Conte. S.

sagittata, L., S., M.

pedata, L. S. M.

primulæfolia, L., S., M.

lanceolata, L., S., II.

blanda, Wild., S., M.

Muhlenhergii, Tor'r., S.

hastata, Michx., S.

pubescens, Ait., S.

var. scabrinscula, $S$.

tricolor, L.

Var, arrensis, M. S.

40. Solea concolor, Ging., S.

XV. Cistacea.

41. Helianthemum Carolinianum, Michx., M.

corymbosum, Michx., M.

42. Lechea major, Michx., M.

minor, Lam., $\mathrm{H}$.

XVI. Droseracece.

43. Drosera filiformis, Raf., M.

longitolia, L., M.

brevifolia, P'ur:h., M. 


\section{Hypericacea.}

44. Ascyrum Crux-Andreæ, L., S.

stans, Michx., S.

microsepalum, 'Torr. \& Gray,

M., S.

45. Hypericum prolificum, L. S.

maculatum, Walt., $\mathrm{M}$.

fasciculatum, L., M.

var. aspalathoides, Chap. M.

galioides, Lam., S.

mỵtifolium, Lam., M.

cistifolium, Lam., M.

angulosum, Michx., M.

pilosum, Walt., M.

mutilum, L., S.

Canadense, L., M. (Mobile) S.

Sarothra, vichx., M. S.

Drummondii, Torr. \& Gray, M.

46. Elodea Virginica, Nutt., S.

petiolata, Pursh., $\mathbf{~}$

XX. Portulacacece.

47. Claytonia Virginica, L., S.

48. Portulaca oleracea, L., S.

49. Sesurium portulacastrum, L.. M.

\section{Caryophyllacece.}

50. Syphonychia erecta, Chap., M.

51. Stipulicida setacea, Michx., M.

5\%. Spergularla media var. macrocarpa, $\mathbf{M}$.

53. Mollugo verticillata, L., S.

54. Spergula arvensis, L., M.

55. Sagina Elliottii, Fenzl., M. S. 
56. Arenaria Pitcheri, Nutt., M. diffusa, Ell., M.

5\%. Cerastium vulgatum, L., S, viscosum, L., M. S. nutans, Raf., M. S.

58. Stellaria pubera, Michx,, S. Media, Smitlı, S.

59. Silene stellata, Ait., S.

Virginica, L., S.

regia, Sims, M.

Antirrhins, L., S. M.

60. Lychnis Githago, L., S.

\section{Malvacece.}

61. Callirrhoe Papaver, Gray M. (Washington County.)

6.2. Sida spinosa, L.

$$
\text { alcæoides, Gray, S. }
$$

rhombifolia, L., M. (Molsile.)

Elliottii, Torr \& Gray, S. M.

(Washington county.)

hispida, S.

63. Abutilon Avicennae, Gaertn. S.

64. Modiola multifida, Móénch., M. S.

65. Hibiscus aculeatus, Walt., M. (Mobile.)

incanus, Wendl., M.

Moscheutos, L. M., (Mobile) S.

militaris, Cav., Dr. Vasser (Cahaba) $M$.

66. Kosteletzkya Virginica, Presl. S.

$\mathrm{XXIV.} \mathrm{Tiliacece.}$

67. Tilia Americana, L., Dr. Denny (Clarke County.) 
68. Corchorus siliquosus, L., M. (Mohile) S

XXV. Camelliace $\alpha$.

69. Stuartia pentagyna, L'Her., M. (Mobile).

XXIX. Linacece.

70. Linum Virginianmm, L., S.

striatum, Walt., II.

usitatissimum, S.

XxX. Oxalidacece.

1 , Oxalis violacea, L. S.

acetocella, L., S.

stïicta, L., S.

XXXII. Geraniacece.

iə. Geranium maculatum, L. S.

Carolinianum, L. S.

XXXIII. Balsaminacece.

73. Impatiens pallida, Nutt, S.

fulva, Nutt., M. S.

XXXIV. Rutacere.

74. Zanthoxylum Ca/olinianum, S., M.

var fruticosum, Gray, M.

75. Ptelea trifoliata L., S.

XXXVII. Anacardiacec.

76. Rhus Toxicodendron

var. quercifolium, Michx. MI.S.

var. 2. radicans, Torr., M. S.

venenata, DC. S., M.

typhina, L., S.

copallina, L., M. S.

cotinoides, Nutt., Buckley. 


\section{8}

XXXVIII. Vitacex.

77. Vitis (cissus) bipinnata,Torr. \& Gray,'S. incisa, Nutt., M. indivisa, Wild., M. S. æstivalis, michx. vulpina, L., M.

78. Ampelopsis quinquefolia, Michx.

\section{Rhamnaceci.}

79. Berchemia volul,ilis, DC., S., M.

80. Sageretia Michauxii, M.

81. Rhamnus lanceolatu:, Pursh. S.

parvifolius, Torr and Gray.

8:2. Frangula Caroliniana, Gray, S. M.

83. Ceanothus Americanus, L., S.

var. b́. Pitcheri.Torr. \& Gray, M.

". intermedius, $\mathbf{x}$.

velistracea.

84. Eumymu A

XL! Staphyleaceœ.

85. Staphylea triwiia, L. S.

XLII. Sapindacece.

86. Cardiospermum Halicacabum, L., S.

87. Aesculus Pavia, L., M. S.

flava, Ait., M. (Montgomery).

parviflora, Walt., S. M., (Wildox Co.)

\section{Aceracece.}

88. Acer rubrum, L., M. S.

dasycarpum, Ehrh. S.

89. Negundo aceroides, $\mathrm{S}$. 


\section{Polygalacece.}

(0) Polygalit eymosil, Wialt. M. (Mohile.)

$$
\begin{aligned}
& \text { lanosa, Ell., U. } \\
& \text { lutea, L. S., M. } \\
& \text { sanguinca L. s. } \\
& \text { uana, DC. S. }
\end{aligned}
$$

Nuttallii, Carer, s.

incirruatil, V. (Molsile.)

Chas manii, Torr. \& (iray, M.

setacea, Miclıx. S.

cruciata, M. (Mol,ile.)

hrevifolia, Nutt., M. (Mobile.)

Hookerii, Torr, \& Gray, ... “

grandiflora, Walt, S., M.

polygama, Walt., M. (Mobile,)

Boykinii, Nutt., s.

rerticillata, L, S., x.

ambigua, M. (Mobile.)

leptostachys, Shuttl., s. (Moh.)

\section{Leguminosce.}

silburder 1. P'upitionucere.

91. Crotalaria sagittalis, L., s.

$$
\begin{aligned}
& \text { oralis, Pursh.. s. } \\
& \text { Purshii, DC., S., .r. }
\end{aligned}
$$

9.). Melilotus officinalis, Willd, s.

$$
\text { parviflorus, } \mathbf{M} \text {. }
$$

93. Lupinns peremis, L., M. (Molile.)

diffisus, Nutt., s. "

villosus, Willd., M. “"

94. Medicago lupuliua, S.

$$
\text { minima M. (Mobile). }
$$

9.5. Trifolium Carolinianum, L., S., M. (Mob.) 
reflexum, L., S.

repens, L.

pratense, L.(escaped from cult.) procumbens, L.

96. Psoralea melilotoides, Michx., var. M, (Mobile).

eglandulosa, Ell., S.

canescens, Michx. M. (Wobile).

97. Petalostemon gracile, Nutt., M.

corymbosum, Michx.

98. Amorpha fruticosa, L., M. (Mobile).

99. Robinia Pseudacacia, L. S.

100. Wistaria frutescens, DC., M. (Mobile).

101. Tephrosia Virginiana, Pers. S.

spicata, Torr. \& Gray, S., M.

ambigua, Curtis, M. (Molile).

hispidula, Pursh., M.

oxobrychoides, Torr. \& Gray,

(Mobile).

chrysophylla, Pursh., M.(Mob.)

10:. Indigofera Caroliniana, Walt., M. (Molo.)

tinctoria, L., (natur. Mobile).

163. Glottidium Floridaum, DC., M. “

104. Seshania macrocarpa, Nutt., M.

105. Astragalus villosus Michx., M.

106. Vicia sativa, L., S. M.

liirsita, Koch, M.

. acutifolia, Ell., M.

Caroliniana, Walt., s.

Ludoviciana, Torr. de Gray, $\mathbf{M}$.

207 . Aeschynomene hispida, Willd., M. (Mol.)

viscidula, Michx., $\mathbf{~}$.

108. Zornia tetraphylla, Michx., s., M. 
109. : tylosanthes elatior, Swartz., S.

110. Lesp dezal repens, Torr. \& Gray, S.

var. procumbens, (Mobile).

violacea, Pers. S.

var. divergens, Chap., s.

var. sessiliflora, Chap., S., M.

var. reticulata, Chap.,M.(Mol).)

var. Stuvei Nutt. S.

capitata, Michx., S., M.

striata,

111. Desmodium nudiflorum, DC., S., M.

acuminatum, DC., M. (Mobile).

viridiflorum, Beck, S.

cuspidatum, Torr. \& Gray M.

rotundifolium, DC., м. (Mol.)

Dillenii, Darl., M.

Canadense; DC: s.

lacvigat um, I) $\mathrm{M}$.

strictum, DC. M.

tenifolinm, Torr d Gr., M. *

Marylandicum, Boott.

ciliare, DC., S. M. (Mol,ile)

rigidium DC. S.

lincatum, DC., м.

11:. Rlı̀neosia minima, DC. м.

tomentosa

var. monophylla, Torr \& Gray, Ell., S. M.

var. erecta, Torr. \& Gray, Ell., M. S.

var. volul,ilis, Torr. \& Gray, M. galactoides, Chap.,M. (Molile).

113. Apios tuberosa, Moench., S. 
114. Phaseolus perennis, Walt., M.

$$
\begin{aligned}
& \text { dirersifolius, Pers., s.. } \\
& \text { helvolus, L. M. (xolbile). }
\end{aligned}
$$

115. Vigna glabra Savi. 1.

116. Erythrina herluacea, L., S.

117. Clitoria Marianna, L. S.

118. Centrosema Virginiana, Benth., S.

119. Amphicarpaea monoica, Nutt., M.

120. Galactia pilosa, Ell., M. S.

$$
\text { mollis, Michx., S., M. }
$$

glabella, Michx., M.

sessiliflora, Torr. \& Gray, $\mathrm{N}$.

1:1. Baptisia leucantha, $\mathbf{M}$.

alba, Brown, s.

lanceolata, Ell., M.

suborder 2. Cuesalpiniea.

1\%.2. Circis Canadensis, L., S.

1:3. Cassia occidentalis, L., S.

ohtusifolia, L., M.

Marṿlandica, L., S.

Chamaecrista, L., S.

biflora, L., s.

nictitans, L., M.

1:4. Gleditschia triacanthos, L., S. monosperma, Walt., S.

sublorder 3. Mimosea.

1\%5. Mimosil strigillosa, Torr. \& Gray, S.

126. Neptunia lutea, Benth., M.

1:27. Desmanthus brachyobus, Benth., M.

l:ss. Schrankia meinata, Willd., s.

angustatil, Torte \& (iray, M. 


\section{Rosaceo.}

1:39. Prumus Chicasal, Michx., s.

Americana, Marsh. umbellata,Ell., M.(Tallapoosa).

(Cerasus) serotina, Elur., S.

(Laurocerasus) Caroliniana, Ait., S.

1:30. Neriusia Alabumensis, Gray, s.

131. Agrimonia Eupatoria, L., S.

parviflora, Ait., M. S.

13:2. Geum all)m, Guelin, M.

133. Sanguisorlua Canadensis, L., S.

134. Waldsteinia fragarioides, Tratt, S.

13.). Potentilla Canadensis, s.

1:36. Fragaria Indica, s.

13i. Rulus villosus, Ait., s.

trivialis, s.

138. Rosa lucida, Elu., s.

rulbiginosia, L., s.

lavigata, Michx., S.

139. Cratagus spathulata, Michx., S.

aprifolia, Miclıx., S.

eoceinea, L., s.

tomentosa, L., s.

glandulosa, Miclıx،, s.

arhorescens, Ell., ..

parvitolia, Ait. s..

1.10. Pyrus corouaria, L., s.

arlutifolia

var. crytlorocarpa, (Mobile).

var. melanocarpa, Dr. Denny.

141. Amelanchier Canadensis

var. listryapium. Torr. \& Gr., S.

14:. Chrysolualanus olılongifolius, Michx., .M. 


\section{Calycanthacew.}

143. Calycantlus floridus, L., S.

latevigatus, Willd., M.

LI. Melastomacece.

14. Rhexia Mariana, L. S.

Viginica, L. S.

stricta, Pursh. S.

glatbella, Michx. S.

ciliosa, Michx. S.

lutea, Walt., M.

LII. Lythracea.

145. Lỵthrum alatum, Pursh., M. (Mohile).

lineare, L. M.

146. Nesai vorticillata, H. B. K., S.

147. Ammania humilis, Michx., S.

148. Cuphea riscossima, Jacq. S.

I.T. Onagraceœ.

149. Gaura filipes, Spach., S.

biennis, L., S.

var. M. (Mobile).

150. Enothera biennis, L. S.

sinuata, L., S.

var. humifusa, Chap., M.

pumila, M.

riparia, Nutt., S.

linearis, Michx., S.

fruticosa, L., S.

151. Epilolium coloratum, Muhl., S.

152. Jussiaa leptocarpa, Nutt., S.

decurrens, DC., S.

graudiflora, Michx., M. 
1.33. Ludwigia alternifolia, L., s.

linearis, Walt., $\mathrm{S}$.

pilosa, Walt., \&.

hirtella, Raf., M.

virgata, Michx., M.

çlindrica, Ell., M.

capitata, Michx., M.

154. Proserpinaca palustris, L., M.

pectinacea, Lam.. M.

LVI. Cactacece.

1.j.). Opuntia rulgaris, Mill, S.

LX. Passifloracece.

151j. Passiflora lutea, L., S.

incaruata, L.. S.

LXI. Cucurbitacea.

159. Melothria pendula, L., M.

1.5. Lagenaria rulgaris.

LXIIII. Crassulace

159. Sedum Nevii, Grar, S.

ternatum, Michx., S.

telephoides, Michx.. S.

160. Penthorum sedoides, L., S.

LXIT. Saxifragacex.

161. Heuchera Americana, L., S.

Curtisii, Gray, Buckley, ex herl,. M.

16\%.). Saxifraga Virginiensis, Michx., S.

Caroliniana, Gray, S.

163. Tiarella Cordifolia, L.. \&.

164. Itea V̈irginica, L.. S.

16.5. Hrdrangea radiata, Walt., s.

quercifolia Bartram. M., s. 
166. Lepuropetalon spathulatum, EH., M.

16i. Decumaria barbara, L., S.

168. Philadelphus grandiflorus, Willd., S.

\section{I.XY. Hamamelacece.}

169. Hanmumelis Virginici, L., S.

10. Liłnidambar Styraciflua, L., S.

\section{LXYI. Umbelliferce.}

171. Hydroctyle unbellata, L., S. interrupta, Muhl., M. rimmuculoides, L., M. repauda, i'ers., M.

17.. Sanicnla Marylandica, L., S.

173. Errngium yucerfolium, Hichx., S.

virgat mm, Lam., s.

1i4. Jeptocamlis divaricatus, DC., M.

echinatus, D)C., M.

17.5. Discoplenm capillacen, Nutt., M.

176. Daneus pnssillus, Michx., s.

17\%. Cicutal maculata, L..

178. Heliosciadinn nodiflorum, Koch., s.

leptoplyllum, DC., S.

1 39 . Simm lineare, Michx., M.

180. Trepocarpus Ethuse, Nutt., M.

181. Tiedmamiat teretifolia, DC., M.

18.2. Archemora rigida, DC., M.

18:3. Charophyllum procmmbens, Lam., S.

Teintmieri, Hook. \& Aru., M.

184. Scindix pecten, I., M.

18.5. Thaspinm auremm, Nutt., M.

trifoliatum, Gray, s.

186. Bupleurum perfoliatum, s.

187. Osmorrhizis herevistylis, DC., s. 
LxVII. Araliace.

188. Aralia spinosi, L., M.

189. Panax quinquefolium, L., S.

LXVIII. Cornacece.

190. Cornus stricta, Lam., S.

florida, L., S.

191. Nrssa aquatica, L., M.

multiflora, Wang., 'S.

uniflora, Walt., S.

\section{DIVISION II.}

Monopetalous Exogenous Plants.

LXIX. Caprifoliacec.

19:2. Lonicera sempervirens, Ait., S.

grata, Ait., S.

193. Triosteum perfoliatum, L., M.

194. Samlucus Canadensis, L., S.

19.5. Vihurnum prunifolium, L., S.

var. ferrugineum, L., S.

nudum, L., S.

scalırellum, Torr. \& Gray, M.

acerifolium, L. II.

LXX. Rubiacece.

I96. Galium Aparine, L., M.

hispidulum, Michx., M.

triflorum, Michx., M. S.

pilosum, Ait., M., S.

var. puncticulosum, Michx., M.

197. Spermacoce glalıra, Michx., S. 
198. Diodia Virginiana, L., S.

$$
\text { teres, Walt., M. }
$$

199. Cephalanthus occidentalis, L., S.

:00. Mitchella repens, L., S.

201 . Oldenlandia coerulea, Gray, S.

var. minor, Torr, \& Gray (Mob.)

rotundifolia, Gray, M.

purpurea, Gray, S.

var. tenuifolia, Gray, S.

augustifolia, Gray, M.

glomerata, Michx., M.

spec.

M.

20:2. Spigelia Marylandica, L., S.

203. Nitreola petiolatil, Torr. \& Gray, M., S.

sessilifolia, Torr. \& Gray, MI.

204. Polypremum procumbens, L., M.

205. Gelsemium sempervirens, Ait., S.

\section{Valerianacece.}

206. Fedia radiata, Michx., M. S.

\section{Compositce.}

207. Vernonia Novehoracensis, Willd., S.

fasciculata

- var. altissima, Torr. \& Gray, S.

angustifolia, Michx., M. S.

208. Stokesia cyanea, L’Her., M.

209. Elephantopus Carolinianus, Willd., S.

tomentosus, L., M. S.

210. Carphephorus Pseudo-Liatris, Cass., M.

coryunl)osil, Torr. \& Gray, S.

211. Ageratum conyzoides, L. M.

21\%. Liatrls elegans, Willd., s. 
squarrosa, Willd., S.

tenuifolia, Nutt.

graminifolia, Willd., S.

spicata, Wllld., S.

gracilis, Pursh., M.

odoratissima, Willd., M.

213. Kuhuia eupatorioides, L., S.

214. Eupatorium purpureum, L., S.

hyssopifolium, L., M.

cuneifolium, Willd., S.

rotundifolium, L., S.

leucolepis, Torr. \& Gray, M.

parviflorium, Ell., M.

teuerifolium, Willd., S,

all)um, L., S.

perfoliatum, L., S.

serotinum, Michx., S.

foniculaceum, L., S.

var. lateriflorum, Torr. \& Gr.,M.

coronopifolium, Willd., S.

215. Mikanea scaudens, Willd., S.

216. Conoclinium coelestinum, DC., S.

217. Sericocarpus conyzoides, Nees., S.

solidagineus, Nees., $\mathbf{2}$

tortifolius, Nees, M.

2)1. Aster concolor, L., S., M.

aduatus, Nutt., M.

patens, Ait., S., M.

virgatus, Ell., M.

undulatus, L., S.

ericoides, I., M.

nultiflorus, Ait., S. 
dumosus, L., S.

var. M.

var. M.

simplex, Willd., S.

219. Erigeron strigosum, Muhl., S.

Canadense, L. S.

Philadelphicum, L., S.

beilidifolium, Muhl., S.

rernum, Torr. \& Gray, S.

2:20. Diplopappus linariifolius, Hook., M.

amygdalinus, Torr. \& Gray, S.

2:21. Boltonia diffusa, Ell., м.

glastifolia, L'Her., м.

22:. Solidago discoidea, Torr. \& Gray, м.

Curtisii, Torr. \& Gray, S.

puberula, Nutt., M.

pulverulenta, Nutt., M.

virgata, Michx., M.

sempervirens, L., M.

patula, Muhl., M.

altissima, L. S.

odora, Ait., S.

amplexicaulis, Torr. \& Gray, S.

Canadensis, L., M.

pauciflosculosa, Michx., M.

lanceolata, L., M.

tenuifolia, Pursh., M.

2:3. Bigelovia nudata, DC., M.

224. Isopappus divaricatus, Torr. \& Gray, S.

2:5. Heterotheca scabra, DC., м.

225. Chrysopsis graminifolia, Nutt. S. 
Mariana, Nutt., S.

gossypina, Nutt. S.

2.2). Baccharis halimifolia, L., M.

228. Pluchea bifrons, DC., M.

fotida, DC., S.

camphorata, DC., M.

229. Polsmnia Uredalia, L., S.

230. Silphium laciniatum, L. S.

compositum, Michx., S.

Asteriscus, I., M.

lævigatum, Ell., s.

231. Chrysogonum Virginianum, L., M.

23\%. Parthenium integrifolium, L., S.

Hỵsterophorus, L. M.

233. Ambrosia artimisiæfolia, L. S.

234. Xauthium strumarium, L. S.

23.5. Eclipta erecta, L., M.

¿36. Bo richia frutescens, DC., M.

23\%. Melanthera hastata, Michx., M.

238. Titragonotheca helianthoides, L. M.

239. Echinacea purpurea, Mœnch., S.

240. liudl, ckia hirta, L, S.

inlyida, Ait., M.

mollis, Ell., S. .

triloba, L. S.

241. Helianthu: Radula, Torr \& Gray, M.

angustifolius, L., M. S.

heterophyllus, Nutt., ..

atrorubens, L., S.

rigidus, Desf., S.

giganteus, L., M.

tomentosus, Michx., S. 
decapetalus, L., S.

liirsulus, Rat., S.

microcephalus, Torr. \& Gray, M.

24:?. Actinon is nudicalis, Nutt., M.

243. Coreopsis aura, Ait., M.

var. leptophylla, $\mathbf{M}$.

var. sul,integra, $\boldsymbol{M}$.

angustifolia, Ait., M.

senifolia, Michx., S.

delphinifolia, Lam., S.

auriculata, L., S.

lanceolata, L., S.

grandiflora, Nutt., $M$.

Drummondii, Michx., M.

244. Bidens frondosa, L., S.

connata, Muhl., S.

chrysanthemoides, Michx., M.

bipinnata, L., S.

245. Spilanthes repens, Michx., S.

Nuttallii, Torr. \& Gray, M.

246. Verbesina Siegesbeckia, Michx., S.

Virginica, L., S.

247. Gaillardia laucenlata, Michx., M. S.

pulchella, Fouger, $\mathbf{M}$.

248. Helenium autumnale, L., S.

tenuifolium, Nutt., S.

249. Leptopoda Helenium, Nutt., M.

fimbriata, Torr. \& Gray, M.

pulserula, Michx., M.

brevifolia, Nutt., $M$.

l) rach ypoda, 'Torr. \& Gray, M.

250. Balwinia uniflora, Ell., M. 
251. Marshallia lanceolata, Pursh., м. S.

$$
\text { latifolia, Pursh., M. }
$$

252. Maruta Cotula, DC., S.

253. Achillea millefolium, L. S.

254. Leucanthemum rulgare, Lam., S.

255. Guaphalium polycephat um, : $\vdots x, \mathrm{~S}$.

$$
\text { purpureum, L., s. }
$$

256. Anteunaria plantaginio iat, Hook., $\therefore$.

55\%. T:macetum vulgare, L., S.

258. Erechthites hieracitoliat, Raf., S.

259. Cacalia attriplicifolia, L., S.

tuberosa, Nutt.. S.

ovata, Walt., $\mathrm{s}$.

lanceolata, Nutt., M.

260. Senecio lobsitus, Pers., S.

vulgaris, L., $\mathbf{M}$.

Elliottii, Torr. \& Gray, S.

61. Cirsium altissimum, Spreng., S.

Virginianum, Michx., S.

horridulum, Michx., M. S.

$\therefore 6$.ะ. Lappa major, Gaert., S.

¿ 03 . Chaptalia tomentosa, Vent., M.

$\approx 64$. Apogon humilis, Ell., S.

z65. Krigia Virginica, Willd., M.

Caroliniana, Nutt., S.

266. Cyuthia Virginlea, Dou., S.

Dandelion, DC., S.

567. Hieracium Gronovii, L., M.

venosum, L., M.

268. Nabalus altissimus, Koch., S.

virgatus, DC., M,

269. Pyrrhoppapus Caroliniauus, LC., S. 
270. Lactuta, elongata, L., S.

2i1. Mulgedium acuminatum, DC., S.

2i. Sonchus oleraceus, L., S.

asper, Vill., S.

\section{Lobeliacece.}

2i3. Lobelia cardinalis, L. S., M.

syphilitica, L., S.

puherula, Michx., M.

leptostachys, A. DC., S.

amœna, Michx., S.

inflata, L., S.

appendiculata, $\mathbf{M}$.

brevifolia, Nutt., M.

Nuttallii, R. \& S., M.

paludosa, Nutt., M.

LXXV. Campanulacece.

274. Campanula divaricata, Michx., S.

Americana, L., S.

275. Specularia perfoliata, A. DC. S.

LXXVI. Ericacece.

276. Gaylussacia dumosa, Torr. \& Gray, M.

frondosa, Torr. \& Gray, M.

27\%. Vaccinium stamineum, L., S.

arboreum, Michx., M.

nitidum, Andr., $M$.

myrsinites, Michx., M.

rar. glaucum, Gray, M.

virgatum, Ait. M.

var. tenellum, Coray, M.

var. parrifolium, Gray, M. 


\section{Elliottii, .s.}

2is. Epigaea repens, L., S., v.

279. Leucothoe axillaris, Don., M.

racemosa, Gray, M.

Catesluaei, Grar, M.

280. Audromeda floribunda, Pursh., M.

phillyreæfolia, Hook., M.

nitida, Bartr., $\mathbf{~}$.

ligustrina, Muhl., M.

281. Oxrdendrum arboreum, DC., S.

282. Kalmia latifolia, L., S.

hirsuta, Walt., M.

2Ł3. Rhododendron nudiflorum, Torr. S., M.

viscosum, Torr., $\mathbf{M}$.

284. Clethra alnifolia, L., S., M.

285. Monotropa uniflora, L., S.

LXXVIII. Aquifoliacece.

2E6. Ilex opaca, Ait., S.

Dahoon, Walt., M.

Dahoon rar. not described, $\mathbf{M}$.

Cassine, L., M.

decidua, Wallt., s.

ambigua, Chap.

Amelanchier. Curtis, $\mathrm{I}$.

glaber, Gray, M.

coriacea, Chap., $\mathrm{M}$.

LXXIX. Styracacece.

287. Styrax Americanum, L., M.

grandifolium, Ait., M.

pulverulentum, Michx.

288. Halesia diptera, L., II. 
totiaptera, L, S.

289. Symplozos tinctoria, L'Her. S.

IXXX. Cyrillacece.

290. Cyrilla racemiflora, Walt., S. 291. Cliftonia ligustrina, Banks, M.

LXXXI. Ebenacea.

292. Diospyros Virginiana, L., S.

LXXXII. Sapotacea.

293. Bumelia lycioides, Gaert., M.

lanuginosa, Pers., M.

LXXXV. Plantaginiacece.

294. Plantago major, L., S.

lanceolata, L., S.

Rugelii, Decaisne, S.

Virginica, L., S.

heterophylla, Nutt., S.

Patagonica.

var. cristata, L., S.

LXXXVI. Plumbaginacece.

295. Statice Caroliniana, Walt., M.

LXXXVII. Primulacea.

296. Steironema, ciliatum, Raf., S.

lanceolatum.

var. angustifolium, Gray, M.

longifolium, Gray, S.

297. Anagallis arrensis, L., M.

298. Centunculus minimus, L. M.

299. Samolus valerandi.

var. Americanus, Gray, S. 
300. Dodscatheon Meadia, L. S.

LXXXViII. Lentibulacere.

301. Utricularia inflata, Walt., M.

filorosa, Walt., M.

subulata, L., If.

cornuta, Michx., M.

302. Pinguicula elatior, Michx., M.

lutea, Walt., $\mathrm{M}$.

LXXXIX. Bignoniacea.

303. Bignonia capreolata, L., S., M.

364. Tecoma radicans, Juss., $S$.

305. Catalpa biguonioides, Walt., M. S.

306. Martynia proboscides, L. S.

XC. Orobanchacece.

307. Epiphegus Virginiana, Bart. S.

XCI. Scrophulariacece.

308. Verbascum Thapsus, L. S.

Blattaria, L., S.

309. Scrophularia nodosa, L., S.

310. Chelone glabra, L., S.

311. Pentstemon pubescens, Solander S.

Digitalis, Nutt., S.

lævigatus, Sol., II.

312. Linaria vulgaris, Miller, S.

Canadeusis, Spreng., S.

Floridana, Chap., J.

313. Mimulus alatus, Ait., S.

314, Herpestis nigresens, Benth., M. S.

chamædroiles, Dr. Dennis.

Vonnieria, Kanth., M. 
315. Gratiola sphærocarpa, Ell., S.

Floridana, Nutt., M.

aurea, Muhl., M.

pilosa, Michx., S., M.

316. Ilysanthes gratioloides, Benth., S.

317. Micranethemum orbiculatum, Michx., M.

Nuttallii, Gray $\mathbf{\text { s. }}$

318. Veronica Virginica, L., S.

var. with 3 leaves in a whorl, $S$.

pregrina, L., S.

arvensis, L., $\mathrm{S}$

319. Buchnera Americina, L. S.

3:20. Seymeria tenuifolia, Pursh., S.

pectinata, Pursh., S.

321. Macranthera fuchsioides, Torr., M. S.

322. Gerardia linifoiia, Nutt., S.

G. (Dasystoma) pedicularii, Benth., M. S.

G. (Otophylla) auriculitı, Benth., Buckley.

aphỵlla, Nutt., M.

purpure:t, L., S.

var. fusciculata, Chap., M.

maritima, Raf., $M$.

Pluckenetti, Ell., M.

var. microphylla, Gray, M.

setacea, Walt., M.

Skinnerianna, Wood, M.

var. pectinata, Gray, S.

pubescens, Benth., S.

laterigata, Raf., $M$.

quercifolia, Pursh., S.

323. Castilleia coccinea, Spreng., S.

3:4. Sehwalbea Americana, L. M.

325. Pedicularis Canadensis, L., S. 
XCII. Acanthace.

3:6. Ruellia strepens, L., S.

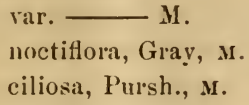

3:). Calophanes humistrata, Nees., M.

humilis, Gray, M.

3०9. — virens, Gray, S.

\section{Verbenaceø.}

330. Verbena officinalis, L., M.

renosa, Giliiss \& Hook, M.

urticifolia, L. S.

Caroliniana, Michx., S.

hastata, L. S.

angustifolia, Michx., S.

bracteosa, Michx., S.

Aubletia, L., S.

331. Lippia nodiflora, Michx., M.

332. Callicarpa Americana, L., S., M.

XCIV. Labiata.

333. Hyptis radiata, Willd., S. M.

334. Mentha piperita, I.., S.

33.). Lycopus Virginicus, L. S.

sinuatus, Ell.

var. intermedius, Chap. S.

Europeus, L., M.

336. Pycnanthenum linifolium, Pursh., S.

incanum, Michx., S.

aristatum, Michx., S.

albescens, Torr. \& Gray, M.

337. Collinsonia Canadensis, L., S. 


\section{anisata, Pursh., M. ovalis, Pursh., M.}

338. Hedeoma pulegioides, Pers., S.

337. Calamintha Caroliniana, Sweet, M.

coccinea, Benth., M.

Nepeta, Link, S.

340. Conradina canescens, Gray.

341. Salvia Chapmani, Gray, Buckley.

urticifolia, L. S.

lyrata, L. S.

coccinea, L. perhaps escaped

from cultivation.

azurea, Lam. S.

34.2. Monarda fistulosa, L., S.

punctata, L., S. M.

343. Blephilia ciliata, Raf., S.

344. Nepeta Cataria, L., S.

34.). Brunella vulgaris, L., S.

346. Scutellaria lateriflora, L., M.

versicolor, Nutt., S.

canescens, Nutt.

var. punctati, Chap., S., M.

serrata, Andr. S.

integrifolia, L., S., M.

pilosa, Michx., S.

parvula, Michx., S.

var. mollis, Gray, S.

galericulata, L., S.

347. Physostegia Virginiana, Benth., S.

348. Marrubium vulgare, L., S.

349. Leonotis nepetæfolia, R. Br., M.

350. Leonurus Cardiaca, L., S., M.

351. Lamium amplexicaule, L., S. 
3 i.:. Trichostema dichotomum, L., S.

lineare, Nutt., II.

333. Teucrium Canadense, L., S.

3.j4. Isanthus coruleus, Michx. S.

\section{$\mathrm{XCV}$. Boraginacece.}

3.5.) Tournefortia heliotropioides, Hook., M. 356. Heliotropium tenellum, Torr., M.

Curassavicun, L., II.

Indicum, L. S.

3.5\%. Cynoglossum Virginicum, L., S.

358. Echinospermum Virginicum, L. S.

359. Liihospermum arvense, L., S.

tuberosum, Rugel, S.

hirtum, Lam., S.

canescens, Lam., S.

360. Onosmodium Virginianum, L., S., M.

361. Myosotis verna, Nutt., S.

\section{Hydrophyllacec.}

36\%. Hydrophyllum Canadense, L., S.

363. Nemophilit microcalyx, Fisch. \& Meyer, S., M.

364. Phacelia bipinnatifida, Michx., S.

Purshii, Buckley, S. parviflora, Pursh., S. pussilla, Buckley, M.

XCVII. Hydroleacec.

36.5. Nama Jamaicensis, L., M. 366. Hydrolea Caroliniana, Michx., S., M.

ovata, Nutt., S. affinis, Gray, M. 


\section{Polemoniacex.}

367. Phlox paniculata, L., S.

$$
\begin{aligned}
& \text { maculata, L., S. } \\
& \text { divaricata, L., S. } \\
& \text { glaberrima, L., S., M. } \\
& \text { var. Laphamii, Wood., S. } \\
& \text { pilosa, L., M. } \\
& \text { Walteri, Chap., M. } \\
& \text { amœna, Sims, M. }
\end{aligned}
$$

368. Polemonium reptans, L., S.

\section{Convolvulacea.}

369. Dichondra repens, Forst., M. 370. Quamoclit coccinea, L. M. S. 361. Ipomoea pes-caprae, Sweet., M. pandurata, Mever, M. sagittata, Cav. M. commutata, R. \& S. M. lacunosa, L., M.

372. Jacquemontia tamnifolia, Gres. M.

373. Calystegia spithamæa, L., S.

$$
\text { sepium, var. rupens, Gray, S. }
$$

374. Breweria (Stylisma) humistrata, Gray, S. M.

375. Cuscuta compacta, Juss., S. decora, Chois. var. pulcherrima, Engel. $\mathbf{M}$. Gronovii, Willd. M.

\section{Solanacea.}

376. Solanum nigrum, L., S.

var. ptycanthum, $\mathrm{S}$. var. nodiflorum, L., S.

\section{Iprnue a a set os afolia} (Batates littoratis Choi) 
Carolinense, L., S.

sisynbriæfolium, Lam. S.

Pseudo-capsicum, L., M.

377. Nicandra physaloides, Gart., S. 378. Physalis obscura, Michx., M.

púluescens, L., S.

lanceolata, Michx., S. M.

angustifolia, Nutt., M.

379. Lycium Carolinianum, Walt., M.

380. Ditura Stramonium, L., S. M.

tatula, L. M.

CI. Gentianacece.

381. Sabhatia macrophylla, Hook., M.

lanceolata, Torr. \& Gray, S.

brachiata, Pursh., s.

paniculata, Pursh., S.

stellaris, Pursh., S.

gracilis, Pursh., M. S.

chlorides, Pursh., M.

gentianoides, Ell., M.

angularis, Pursh., S.

calicosa, Pursh., S.

Boykinii, Gray, S.

38:). Gentiana Elliotti, Gray, u.

ochroleuca, Froel., S., M.

Saponaria, L., . I.

383. Frasera Carolinensis, Walt., Dr. Denny.

384. Bartonia tenella, Muhl., M.

verna, Muhl., м.

385. Obolaria Virginica, L., S.

386. Limnanthemum trachyspermum, Gray, M. 
CII. Apocynacece.

387. Apocynum cannahinum, L., M.

androsæunifolium, L. S.

388. Trachelospermum difforme, Gray, S.

389. Amsonia Tabernæmontana, Walt., S.

angustifolia, Michx., S.

\section{Asclepiadacece.}

390. Asclepiodora vlridis, Gray, M.

391. Asclepias tuberosa, L., S.

var. decumbens, M. paupercula, Michx., M.

obtusifolia, Michx., v.

variegata, L., S.

perennis, Walt., S.

verticillata, L., S.

- angustifolia, Ell., M.

Michauxii, Decaisne, M. amplexicaulis, Michx. $\mathbf{y}$.

392. Acerates paniculata, Decaisne, S.

viridiflora, Ell., м.

longifolia, Ell., M.

393. Ens!enia a!hida, Nutt., S.

394. Sentera palustris, Gray, M.

matitima, Decaisne, s.

395. Gonololus lirsutus, Michx., S.

Baldwiniensis, Sweet, Buckley.

CIY. Oleacece.

396. Forestiera acuminata, Poir., M.

ligustrina, Poir., S.

397. Chionanthus Virginica, L., S., M.

398. Olea Americana, L., M. S. 
39!). Fraximus Americana, L., Dr. Denny, S.

var. microcarpa, Gray.

plat yearpa, Michx., S.

CV. Aristolochiacere.

400. Asarum Virginicum, L., S.

arifolium, Michx., S., M.

401. Aristolochia serpentaria, L., S., M.

tomentosa, Sims, Dr. Denıy.

CVI. Nyctaginacece.

40:2. Oxybaphus albidus, Sweet., M.

403. Boerhaaria erecta, L., S., . I.

CVII. Phytolaccacece.

404. Phytolacca decandra, L. S. M.

\section{CriII. Chenopodiacea.}

405. Chenopodium Boscianum, Moq., M.

album, L., M. S.

Botrys, L., S.

Anthelminticum, L., S.

406. Obione arenaria, Moquin, s.

40i. Salicornia ambigna, Michx., M.

408. Chenopodina maritima, Michx., si.

409. Salsola Kali, L., M.

CIX. Amarantacece.

410. Anarantus chlorostachỵs, Willd., .r.

hylbridus, L. M.

spinosus, L. S.

411. Acnida camnabina, L., M.

41\%. Alteranthera Achyrantha, R. Br., S., M.

413. Frolichia Floridana, Moquin, S. MI. 


\section{Polygonacea.}

414. Rumex crispus, L., S.

obtusifolius, S., M.

Floridanus, L., M.

sanguineus, L., S.

Acetosella, L., S.

hastatulus, Baldw., S.

415. Polygonella parvifolia, Michx., Buckley.

gracilis, Meisner, $\mathbf{M}$.

Meisneriana, Shuttle., Rugel.

416. Polygonum incarnatum, Ell., ...

Pennsylvanicum, L., .r.

Persicaria, L., S.

hydropiperoides, Michx., M.

acre, Kunth. S.

setaceum, Baldw., S., M.

a viculare, $\mathrm{L}$. $\mathrm{S}$.

var. erectum, Chap., S.

Virginianum, L., S.

sagittatum, L., S.

dumetorum, L., S., M.

41\%. Brunnichia cirrhosa, Banks, S... M.

CXI. Lauracece.

418. Persea Carolinensis, Nees., M.

rar. palustris, Chap., $\mathbf{M}$.

419. Sassafras officinale, Nees., S.

4\%0. Benzoin melissafolium, Nees., Buckley.

CXI. Thymeleacer.

421. Dirca palustris, L., Dr. Denny.

CXIV. Loranthacea.

4.:. Phoradendron flavescens, Nutt., M., S. 


\section{7}

CXV. Saururacea.

423. Saururus cernuus, L. S. M.

CXVI. Ceratophyllacece.

4:24. Ceratophyllum demersum, L., M.

CXVII. Callitrichaceee.

425. Callitriche heterophylla, Pursh.

var. hrevifolia, $M$.

var. terrestris, M.

CXIX. Euphorbiacere.

4 (6. Euphorbia corollata, L. S. M.

rar. anrostifolia, Ell., u.

telephioides, Chap., M.

hypericifolia, L. S.

pilulifera, L., M.

maculata, L. S. is.

cordirolia, Ell., M.

polygonifolia, L., M.

obtusata, Pursh., Buckley.

Peplus, L., M.

commutata, Engelm. S.

4:27. Stillingia sylvatica, L., S., M.

lingustrina, Michx., M.

sebifera, Michx., M.

428. Acalypha Virginica, L., S.

var. gracilens, Gray, S.

Caroliniana, Walt., S., м.

429. Cnidoscolus, stimulosus, Gray, S., M.

430. Tragia innocua, Walt., M.

urticifolia, Michx., S., м.

431. Croton argyranthemum, Michx., M.

capitatum, Michx., S. 
Texcnese, Moe. $\mathbf{m}$.

glandulosum, L., S., M.

monanthogynum, Michx., S.

43:. Ricinus communis, L.

433. Phỵllanthus Carolinensis, Walt., M. S.

434. Crotonopsis linearis, Michx., M.

CXX. Empetracece.

435. Geratiola cricoides, Michx., M.

CXXI. Batidacece.

436. Batis maritima, L., M.

CXXII. Urticacea.

437. Urtica Chamædryoides, Pursh., S.

purpurascens, Nuts., S.

438. Pilea pumila, Gray, M., S.

439. Berhmeria cylindrica, Willd.

CXXIII. Cannabinacea.

40. Humulns Lupulus, L., S.

CXXIV. Moracea.

441. Morus rubra, L., S.

$$
\text { alba, L., S. }
$$

442. Broussonetia papyrifera Vent.

443. Macluria aurantiacea, Nutt., S. M.

CXXV. Ulmacea.

444. Ulmus fulva, Michx., S.

Americana, Dr Denny, S.

alata, Michx., M. S.

445. Planera aquatica, Gmel., M.

446. Celtis occidentalis, L., S.

Mississippiensis, M. 
CXXVI. Platanacea.

447. Platauus occidentalis, L. S. M.

CXXVII. Juglandacea.

448. Carya alba, Nutt., M. S.

oliveformis, Nutt., S. M.

tomentosa, Nutt., м. S.

aquatica, Nutt., M.

amara, Nutt. M. S.

449. Juglans nigra, L., S.

cinerea, L., S.

CXXVill. Cupuliferæ.

450. Quercus Phellos, I., S., M.

arenaria, s.

virens, Ait., .M.

maritima, Willd..

dentata, M.

cinerea, Michx., м.

var. nondescript (Citronelle.)

var. pumila, Michx. M.

aquatica (Catesby), S., M.

nigra, L., S., M.

Catesbaei, Michx., M.

tinctoria, Bartr., S.

coccinea, Wang., $M$.

rubra, L. S.,

falcata Mlchx., S., M.

obtusiloba, Michx., S.

alba, L., S., M.

lyrata, Walt., S.

bicolor, Walt., S.

Prinus, L. S. 
Pr. acuminata (Q.

Castanea), Willd., s. pumila, Walt., $\mathrm{S}$.

4il. Castanea resca, L. S.

$$
\text { pumila, Michx., M. S. }
$$

4.5.2. Fatgus ferruginea, Ait., S.

453. Corylus Americana, Michx., Walt., S.

454. Carpinus Amcricana, Michx., S., M.

455. Ostrya Virginica, Willd., S., Dr. Denny.

CXXIX. Myricacec.

456. Myrica cerifera, L., M.

$$
\begin{aligned}
& \text { var. media, Michx., M. } \\
& \text { var. pumila, Michx., M. } \\
& \text { inodora, Bartr., M. } \\
& \text { CXX. Betulacere. }
\end{aligned}
$$

457. Betula nigra, L., S.

458. Alnus serrulata, Ait., S., M.

CXXXI. Salicacec.

459. Salix nigra, Marsh., M. 460. Populus grandidentata, Michx., S.

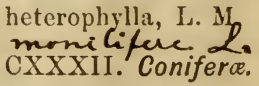

461. Pinus tada, L., S., M.

$$
\text { australis, S., H. }
$$

Pinus glatr Wilh mitis, Michx., S.

Elliottii, $\mathbf{M}$.

serotina, Michx., Curtis.

462. Juniperus Virginiana, L., S., M.

463. Cupressus thyoides, L. M.

464. Taxodium distichum, Rich., S. M. 
CIASS II.

\section{Monocotylodonors or Endogenors}

Plants.

CXXXIV. Palmoe.

46.5. Salual serrulata, R. \& S., M.

Adansonii, Guerns., S., M.

CXXXV. Aracea.

466. Arisema triphyllum, Torr., S.

Dracontium, Shott, S.

46ĩ. Peltandra Virginica, Raf. S.

468. Xanthosoma sagittifolium, Schott, M.

469. Orontium aquaticum, L. S.

470. Acorus calamus, L. S.

CXXXVI. Lemnacea.

4i1. Lemna polyrhiza, L., M.

CXXXVII. Typhaceœ.

47.. Typha latifolia, L. S. M.

473. Sparganium simplex.

- tar aretrotladiuas, Gray, S.

CXXVIII. Naiadaceco.

474. Naias flexilis, Rostk., M.

475. Zannichellia palustris, L., M.

476. Ruppia marima, L., M.

477. Potamogeton fluitans, Roth., M.

perfoliatus, L., M.

hybridus, Michx., M. 
CXXXIX. Alismaceœe.

478. 'Triglochin triandrum, Michx., M.

479. Alisma Plantago, L., S.

4\$0. Sagrittaria talcata, Pursh., M.

variabilis, Engelm., M. S.

simplex, Pursh, M.

natans, Michx.

var. loratta-pusilla, Pursh., M.

CXL. Hydrocharidacea.

481. Vallisneria spiralis, L., M.

\section{Burmanniacee.}

48:. Burmannia biflora, L., M.

capitata, Chap., $\boldsymbol{\text { s. }}$

483. Apteria setacea, Nutt.. M.

\section{Orchidaceœ.}

484. Microstylis ophioglossoides, Nutt., S.

48.). Epidendrum conopseum, Ait., M.

686. Tipularia discolor, Nutt., S., M.

48\% - Bletia aphỵlla, Nutt., S.

488. Calopogon pulchellus, R. Br. S.

parviflorus, Lindl., M.

45\%. Pogonia ophioglossoides, Nutt., S., M.

divaricata, R. Br., M.

verticillata, Nutt., S.

490. Habenaria (Gymnadenia) flara, Lindl.,S.

" tridentata, Lindl., S.

" nivea, Gray \& Fn-

$$
\text { gelm., M. }
$$

(Platanthera) ciliaris $\mathrm{R} . \mathrm{Br}$. S.

" blephariglottis, Hk. S. 
" cristata, R. Br. M. S.

" lacera, Gray, S.

repens, Nutt., M.

Michauxii, Nutt., S.

4!). Spiranthes cernua, Rich., S.

tortilis, Willd., S.

graminea, Lindl.

var. Walteri, M.

gracilis, Bigel, $\mathbf{~ r . ~}$

brevifolia, Chap., $\mathrm{s}$.

49:. Ponthieva glandulosa, R. Br., M. S.

\section{Cannacer.}

493. Canna flaccida, Roscoe, 3.

CXLIV. Amaryllidacee.

494. Amaryllis Atamasco, L., S.

495. Pancratium rotatum, Ker., M.

occidentale, Leconte, S.

496. Crinum Americanum, L., M.

497. Agave Virginica, L. S.

498. Hypoxis erecta, L. S.

CXLV. Hamodoracea.

499. Lachnanthes tinctoria, Ell., II.

500. Lophiola aurea, Ker., M.

501. Aletris farinosa, L., S., M.

aurea, Walt., M.

CXLVI. Bromeliacec.

50\%. Tillandsia usneoides, L.

CXLVII. Iridacee.

503. Iris verna, L., M.

versicolor, L. M. 


\section{4}

cristata, Ait., S.

504. Sisyrinchium Bermudiana, L. S.

var. mucronatum, Gray, M.

var. alhidum, Gray, S.

CXLIIII. Dioscoreacer.

50.). Dioscorca villosa, L., S.

\section{Smilacea.}

506. Smilax rotundifolia, L., S., M.

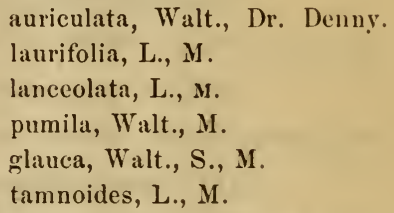

50\%. Coprosmanthus herbaceus, Kunth., S.

508. Trillium sessile, L. S.

discolor, Wray, M.

cernuum, L., S.

509. Medeola V'iginica, L., S.

\section{Roxburghiaceœ.}

510. Croomia panciflora, Torr., S.

\section{Liliaceœ.}

511. Polygonatum biflorum, Ell., S.

j):. Smilacina racemosa, Desf., S.

503. Allium ('anadense, Kalm., S.

$$
\begin{aligned}
& \text { mutabile, Michx., S. } \\
& \text { striatum, Jacq., S. M. }
\end{aligned}
$$

514. Scilla Fraseri, Gray, S.

515. Erythronium Americanum, Smith, S.

516. Lilium superhum, L. S. 
var. Carolinianum, Chap., M.

Catesbatei, Wallt., M.

SIi. Yucea filamentosa, L., S., M.

aloifolia, L., S.

CLII. Melanthace

.j1. Urularia perfoliat:a, L., S.

sessilifolia, L., S.

i19. Melanthium Virginicum, L., M.

5:20. Zigadenus glaherrimus, Miclıx., M.

5:21. Stenanthium angustifolium, Gray, S.

5:2.). Amianthium muscetoxicum, Gray, S.

5::3. Chamelirium luteum, Gray, S.

524. Tofieldia pubens, Ait., M.

CLIII. Juncacece.

5:5.5. Luzula campestris, DC., S.

5:26. Juncus effusus, L., S.

setaceus, Rostk., S.

Roemerianus, S. Schul). M.

tenuis, Willd., S.

var. B. M.

dichotomus, Ell., M.

bufonius, L. S.

var. fasciaclaris, Engelm., M.

acuminatus.

var. legitimus, Engelm., M.

scirpoides, Lam. S.

var. polycephalus, M.

var. macrostemon, Gray, M.

marginatus, Rostk., S.

var. biflorus, Engelm., M.

repens, Michx., M. 
CLIV. Pontederiacse.

5.2i. P'ontederia cordata, L., M.

.): sithollera graminea, Willd., s.

CIT. Commelynacece.

5:29. Commelyna Cajemnonsis, Rich., M. S.

erecta, L., M.

Virginica, L., S., M.

530. Tradescantia Virginica, L. S. M.

CLVI. Mayacacer.

531. Mayaca Michauxii, Schott, \& Endl., M.

CI.TII. Xyridacea.

j3:. Xyris flexuosa, Muhl., M. s.

ambigua, Beyr., $\boldsymbol{M}$.

Elliottii, Chap., $\mathbf{x}$.

Caroliniana, Walt., M.

iridifolia, Chap., $\mathbf{r}$.

torta, Smith, M.

tenuifolia, Chap., M.

Baldwiniana, R. \& S., M.

CLYIII. Eriocaulonacea.

533. Eriocaulon decangulare, L., S.

gnaphalodes, Michx., M.

534. Lachnocaulon Michauxii, Kuntlı., м.

CLIX. Cyperacea.

535. Cyperus flarescens, L., S.

rivularis, Kunth., S., M.

Nuttallii, Torr., $\mathbf{M}$.

microdontus, Torr., $\mathrm{M}$.

stenolepis, Torr., M. 


\section{7}

Michauxianus, Schultes, s.

repens, Ell., $\mathbf{~ r .}$

rotundus, L., S., M.

articulatus, L. M.

Haspan, L., S., м.

Le'contii, Torr... $\mathbf{~ r . ~}$

dissititlorus, Torr., M.

virens, Michx., S., M.

regetus, Willd., S.

compressus, L. M.

filiculmis, Tahl., M.

Grayii, Torr., M.

ovularis, Torr., M. S.

retrofractus, Torr. M. S.

Baldwinii, Torr., S.

5330. Kyllingia pumila, Yichx., M,

sesquiflora, Torr. S.

5.3i. Dulichium spathaceum, Rich., M., S.

538. Hemicarpha subsquarrosa, Nees., M.

539. Lipocarpha maculata, Torr., S.

54). Fuirena scirpoidea, Vahl., M.

squarrosa, Michx., S.

var. hispida, Chap., M.

541. Elcocharis equisetoides, Torr., M.

cellulosa, Torr., $\mathbf{M}$.

tuberculosa, R. Br., S.

prolifera, Torr., S.

allida, Torr., $\mathbf{M}$.

tenuis, Schultes, S., M.

microcarpa, Torr., M.

olstusa, Schultes, S.

acicularis, R. Br., M.

pygmaa, Torr., $\mathbf{M}$. 


\section{8}

Baldwinii, Torr., M.

54:. Scirpus pungens, Vahl, м. valiclus, Vahl, M.

leptolepis, Chap., $\mathbf{\text { I. }}$

maritimus, L., $\mathbf{~}$.

Eriophorum, Michx., м., S.

lineatus, Michx., M.

Olneyi, Gray, м.

543. Fimbristylis spadicea.

var. castaneus, Gray, $\mathbf{~}$

var. puberuta, Michx., M.

544. Trichelostylis autumnalis, Chap., S.

j4.). Isolepis ciliatifolia, Torr., M.

stenophylla, Torr., M.

546. Rhyncospora plumosa, Ell., M.

var. intermedia, Chap., $\mathbf{M}$.

oligantha, Gray, M.

rariflora, Ell., M.

Torreyana, Gray, м.

cymosa, Nutt., M.

var. glohularis, Chap., M.

compressa, Gray, Buckley.

microcarpa, Bald., M.

inexpansa, Vahl, M.

Eliiottii, Dietr., M*

caduca, Ell., M.

miliacea, Gray, M., S.

Grayii, Kunth., M.

ciliata, Vahl, M.

megalocarpa, Gray, M.

fascicularis, Nutt., M.

var. distans, Chap., M.

filifolia, Gray, M. 
pallida, C'urtis, M.

gracilenta, Gray, M.

glomerata, Vahl, M., s.

cephilantha, (iray, M.

pusilla, Chap., $\mathbf{~}$.

5.17. Ceratoschoenus corniculatus, Nees, S.,M.

54. Dichromena latifolia, Bald., M.

leucocephala, Michx., S.

549. Cladium elfusum, Torr., $\mathbf{~}$.

5.i). Seleria triglomerata, Michx., M.

oligantha, Fll., S.

Elliottii, Chap., $\mathbf{~}$.

laxis, Tors., M.

pauciflora, Muhl., M.

var. glabra, Chap., м.

Michauxii, Chap., $\mathbf{M}$.

55l. Carex (Vignea).

bromoides, Schk., M.

rulpinoidea, Michx., M.

Crus-corvi, Shuttl., M.

cephalophora, Muhl., M., S.

stellulata.

var. conferta, Chap., $\mathbf{M}$.

scoparia, Schk.

straminea, Schk., M.

foenea, Muhl., M. S.

Muhlenbergii, S.

5.52. Carex (proper).

polytrichoides, Muhl., S., M.

virescens, Muhl., M.

dasycarpa, Muhl., M.

laxiflora, L. S.

var. striatula, Chap., $\mathbf{~}$. 
styloflexa, Timekley, $\mathrm{N}$.

ilaucseens, Eil., S. M.

Cherokeensis, Schk., m.

venusta, Dew, M.

tentatulata, Muhl., M. S.

lupulina, Muhl., s.

folliculata, L., $\mathbf{M}$.

turgescens, Torr., S.

Elliottii, Schw. \& Torr., M.

intumescens, Rudge, M. S.

CLI. Gramineœ.

553. Leersia Virginica, Willd., м.

oryzoides, Swartz, M.

5.)4. Zizania miliacea, Michx., M. aquatica, L., M.

5.5.5. Hydrochloa Carolinensis, Beauv., M.

5j(i. Alopecurus geniculatus, L., S.

pratensis, M.

557. Phleum pratense, L., м.

558. Sporoloolus Indicus, Brown, S., M.

junceus Kunth., s.

5.59. Vilfa aspera, Beaur., $\mathbf{~}$.

vaginaflora, $\mathbf{~}$.

560. Agrostis perennans, Gray, M.

scabra, Wllld., S.

albi, L., M.

arachnoides, Ell., $\mathbf{M}$.

571. Cimma arundinacea, L. S. M. j(i). Muhlenbergia Mexicana, Trin., M.

diffusa, Schreb., M. sylvatica, Willd., M. capillaris, Kunth., M. S. 
trichopodes, Chap., W.

olios. Brachyclytrum aristatum, Peaur., M.

.)64. Caldmagentostis Nuttallii, S.

i(i.). Aristida gracilis, Ell., M.

lamata, Poir., S.

purpurasceus, Poir., M.

virgata, Trin., $\mathbf{M}$.

var. palustris, Chap., M.

spicitormis, Ell., M.

¿66. Spartiua juncea, Willd., M.

polystachya, Willd., M.

glabra, Muhl., M.

567. Gymnopogon racemosus, Beauv., M.

brevifolius, Triu., M.

568. Eustachys petriea, Desv., M.

5(i). Cynodon dactỵlon, Pers., s.

5). Ctenium Americaum, Spreng., M.

5i1. Dactyloctenium Agyptiacum, Willd., S.

572. Eleusine Indica, Grert., S.

5\%3. Leptochloa mucromata, Kunth., S. polystachya, Kunth., M.

574. Tricuspis sesslerioides, Tort., S.

ambigua, Clıap., $\mathbf{~}$.

cornuta Cray, M.

purpurea, Gray, м.

575. Dactylis glomerata, L., S.

576. Eatonia oltusata, Gray, м.

Pennsylvanica, var. filiformis, Clıap., M.

5) 7 . Melica mutica, Walt., S.

5\%8. Glyecria nervata, Trin., M.

5\%9. Arundinaria gigautea, Chap., $\mathrm{S}$.

tecta, Muhl., M. 
580. Brizopyrum spicatum, Hook., s.

581. Joa annua, L. S. M.

cristata, Walt., $\mathbf{M}$.

compressa. L., M.

alsodes, Gray, S. M.

flexuosa, Muhl., M.

praten sis, L., M. S.

582. Eragrostis reptans, Nees., M.

pocoides,

var. magastachya, Gray, S.

ciliaris, Link., M.

Purshii, Schrad., M.

conferta, Trinn., $\mathbf{~}$.

tenuis, Gray, M.

capillaris, Nees., S.

nitida, Chap., $\mathbf{~ r . ~}$

pectinacea, Gray, M.

var. refracta, Chap., $\mathrm{M}$.

583. Festuca tenclla, Willd., S.

Myurus, L., M.

parviflora, Ell., M.

unioloides, Willd., S., M.

584. Bromus ciliatus, L., M.

secalinus, L., S.

Scraderi, $\mathbf{M}$.

585. Uniola latifolia, Michx., S.

paniculata, L., M.

gracilis, Michx., S.

nitida, Baldw., M.

586. Phragmites communis, Trin., M.

587. Elyıus Virginicus, L., M.

striatus, Willd., M.

588. Gymnostichum Hystrix, Schrcb., M. 
5s?. Lolium perenne, L., S.

590. A ira caryophyllea, L., M.

5.)1. Hordeum pratense, L. M.

59:). Danthonia sericsa, Nutt., M.

spicata, Beaur., M.

5!93. Arrhenatherum avenaceum, Beauv., M.

59). Avena fitua, L., s.

59.5. Phalaris intermedia, Bosc., M. S.

59)6. Holcus lanatus, L., M.

59)7. Paspalum fluitans, Kunth., s.

Walteri, Schultes., M.

distichum, L., M.

Digitaria, Poir S.

platycaule, $\mathrm{S}$.

praecox Walt. S. M.

læve, Michx., M. S.

Floridanum Michx., is.

racemulosum, Nutt., $\mathbf{M}$.

undulatum, Poir., S.

ciliatifolium, Michx., M. S.

598. Panicum (Digitaria).

sanguinale, L., S.

filiforme, L., M.

villosum, Ell., M.

(Panicum proper)

gibbum, Ell., $\mathbf{\text { M. }}$

Curtisii, Chap., M.

hians, Ell., M.

anceps, I., M.

divergens, Muhl. M.

virgatum L., S.

amarum, Ell., M.

proliferum Lam., M. 


\section{4}

verrucosum, Muhl, M.

litifolium, L. S.

clandestinum, L., м.

scoparium, I., S. M.

pauciflorum, Ell., ...

viscidum, Ell., S., M.

microcarpum, Muhl., S.

dichotomum, L., S., M.

depauperatum, Muhl., м.

ignoratum, Kunth., M.

Crus-galli, L., S., M.

colonuin, L., II.

livtellum, L., M.

lepens, L., $\mathbf{M}$.

5.99. Setaria glauea, Beaur., м.

var. lavigata, S.

Italica, I., M.

(60). Cenchrus echinatus, L., M.

tribuloides, L., $\mathbf{M}$.

01. Stenotaphrum Americanum, Schrauk.,M.

(6):2. Rotthollia lugosit, Nutt., M.

corrugata, Baldw., s.

(6):3. Tripsacum dactyloides, L., S.

(i) 4. Andropogon tener, Kunth., м.

scoparius, Michx., S.

furcatus, Muhl., S.

tetrastaclyus, Ell., M.

Elliottii, Chap., M.

Virginicus, L. S.

macrourus, Michx., M.

(60.). Erianthus alopecuroides, Ell., S., M.

var. contortus, Chap., S.

var. Hreviluarbis, Chap., $\mathbf{M}$. 
606. Sorghum avenaceum, Chap., S., м. nutalls, Gray, S., м.

607. Luziole Alabamensis, Clap.. Beaumont.

\section{Equisetacece.}

608. Eq̨uisetum laevigatum, Braun, S.

\section{Filices.}

609. Polypodiun vulgare, L., S.

$$
\text { incanum, Swartz, M. S. }
$$

610. Pteris serrulata, L., S., M.

aquilina.

var. caudata, Chap., S.

611. Pellaa atropurpurea, Link., Chap., S.

61\%. Cheilanthes Alabamensis, Kunze, S.

restita, Swartz, S.

tomentosit, Link, S.

613. Adiantum Capillus-Teneris, L. S.

pedatum, L. S.

614. Woodwardia angustifolia. S.

Virginica, Willd., s.

615. Camptosorus rhizophryllus, Link, S.

616. Asplenium pinnatifielum, Nutt., S.

chenoides, Scott, S.

ebeneum, Ait., S. M.

var. incisum, M. (Montgomery).

parvulum, Mart. \& Gal. S.

Trichomames, L. S.

montanum, Willd., S.

Ruta-muraria, L., S.

Filix-formina, Bernh. S.

617. Cystopteris fragilis, Bernh., S. 618. Phegopteris hexagonoptera, Fee. S.

polypodioides, Fee. S. 
(i19. Aspidiun Thelypteris, Swatz, s.

Norehoracense, Willd., s.

patens, Swartz, s., M.

marginale, Swartz, S.

acrostichoides, Swartz., S.

(i:20. Onoolea sensibilis, I., S.

621. Woodsia obtusa, Torr., S.

(i):.). Dicksonia punctilobula, Kunze, $\mathrm{s}$.

(6:23. Trichomanes Petersii, Gray, S.

radicans, Swartz, S.

6:24. Osmunda regalis, L., S., M.

cinnamomea, L., S., M.

(j2:). Botrychium ternatum, Swartz, м.

var. lunarioides Willd., M.

Virginicum, Swartz, S.

6:26. Ophioglossum bulbosum, Michx., M. S. nudicaule, L.

CLXIII. Lycopodiacece.

627. Lycopodium alopecuroides, L., M.

inundatum, $\mathrm{L}$.

var. attenuatum, $\mathbf{s .}$

Carolinianum, L., M.

cernuum, L., M.

628. Selaginella apus, Spring., S.

CLXIV. Hydropterides.

629. Azolla Caroliniana, Willd., M. 


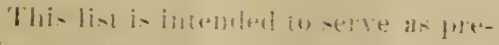
liminary io a emmple ie eatalegure of the Florat of Alabamat to be phiblishel here-

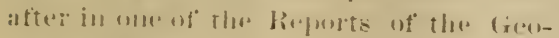
logical survery.

It is desired that these to whom this

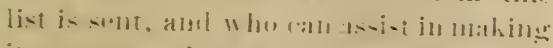

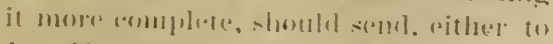

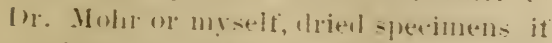
possible ot lattive plants olserrend by

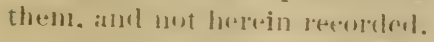

$$
\begin{aligned}
& \text { FI GFNE A. SMITH, } \\
& \text { Thuralonsil, }
\end{aligned}
$$

(s) dlit. 
